\title{
Optimal Location of IPFC for Improving Power System Performance Using Cuckoo Search Algorithm
}

\author{
Dr. Arul $\mathrm{S}^{1}$, Chellaswamy $\mathrm{C}^{2}$ \\ ${ }^{I}$ Department of Electronics and Communication Engineering, Jeppiaar Institute of Technology, Chennai, India. \\ ${ }^{2}$ Department of Electronics and Communication Engineering, Rajalakshmi Institute of Technology, Tamil Nadu, \\ India,
}

\begin{abstract}
This paper presents a new scheme based on cuckoo search algorithm (CSA) for enhancing the performance of interline power flow controller (IPFC) under multiline transmission for reducing the transmission line congestion to a great extent. Optimal placement of IPFC is done by subtracting line utilization factor (SLUF) and CSA-based optimal tuning. The multi objective function consists of active power loss, security margin, bus voltage limit violation and capacity of installed IPFC. The multi objective function is tuned by CSA and the optimal location for minimizing the congestion in transmission lines is obtained. The simulation is performed using MATLAB for the case study using an IEEE 30-bus test system. The performance of CSA has been studied under different loading conditions and compared with two other optimization techniques such as particle swarm optimization (PSO) and differential evolution algorithm (DEA). The result shows that the proposed CSA outperforms the other two methods and it best suits the power system security.
\end{abstract}

Keywords : congestion management; cuckoo search algorithm; Interline power flow controller (IPFC); optimal location; power system; subtracting line utilization factor

\section{Introduction}

There is an enormous increase in power transaction due to power system renovation and different factors such as environmental, right-of-way and high cost, which forms the hurdle for the expansion of power transmission network. With the advancement in flexible Ac transmission system (FACTS), several innovative concepts turn the system into more flexible and have control over power flow without altering the generation schedule. Optimal location identification and allocation of FACT devices improve various parameters of the system [1], [2]. FACT is based on power electronics and other stationary tools which control one or more parameters of AC transmission system thereby increasing the power transfer capability and controllability [3]. Various FACT tools has been used for this purpose such as static Var compensator (SVC), static synchronous compensator (STATCOM), static synchronous series compensator (SSSC), thyristor controller series capacitor (TCSC), unified power flow controller (UPFC) and interline power flow controller (IPFC) [4].

IPFC combines two or more FACT controllers in series and can control power flows of a group of lines and sub-networks. On the other hand the UPFC can control power flow of single transmission line only. The IPFC also has the capability to directly transfer real power between compensated lines and transfer power demand from over loaded to under loaded lines [5], [6]. To solve power system state estimation problem Taguchi differential evolution algorithm is used. For improving the accuracy and reliability of state estimation problem the positive properties of Taguchi method is combined with the differential evolution [7]. Application of differential evolution algorithm for transient stability with different constrains to get optimal power flow has been introduced by Cai et al [8]. A modified differential evolution algorithm with fitness sharing for increasing the stability, reduce overload and voltage violations of power system [9]. A particle swarm optimization (PSO)based algorithm is used to estimate exact location and sizing of unified power flow controller to perform congestion management. The impact of load variations, system reliability and congestion cost of the system has been studied [10]. Automatic human motion tracking in video sequences using PSO technique is proposed by Sanjay et al [11]. Computer vision and pattern recognition is used to identify the motion of human and high search space is used for high variability in human appearance.

CS algorithm is the nature inspired algorithm for optimization heuristics to solve difficult optimization problems. The obligatory brood parasitism with levy flight is a unique behavior of CS algorithm [12]. Comparison of several FACT devices using CS algorithm for three unequal areas of thermal systems has been studied in [13]. Distributed network reconfiguration for power loss minimization, load frequency control, voltage profile improvement for nonlinear interconnected power system using CS algorithm has been studied [14], [15]. Multi-objective short-term scheduling and non-convex economic dispatch considering system characteristics including valve-point effects, multiple fuels, prohibited zones and power loss using CS method has been studied [16], [17]. Line utilization factor (LUF) is used to determine the percentage of loading by 
considering real and reactive power flowing in the line [18]. LUF is used for estimating congestion of a single transmission line whereas IPFC is connected to multiple transmission lines. Hence LUF is not sufficient for estimating the location of IPFC

In the above survey, no generalized approach is available for placing any FACT devices. Number of methods has been formalized for finding the optimal location for a particular type of FACT device. In this paper, the optimal location of IPFC is estimated by subtracting LUFs between two lines and the line pairs are ranked based on congestion and the priority has been allotted. The optimal tuning of IPFC is carried out by formulating a multi objective function. The multi objective function includes minimization of load voltage deviation, Maximization of VSM and reduction of active power loss. Optimal tuning is done to reduce loss and which in turn leads in reduction of transmission line congestion. The performance of CSA has been compared with two other optimization techniques such as particle swarm optimization (PSO) and differential evolution algorithm (DEA) under IEEE 30-bus test system. The performance of proposed method is tested under different loading conditions. The remainder of this paper is organized as follows: section 2 describes the modeling of IPFC; section 3 describes optimal sizing of IPFC, section 4 explains about optimal allocation of IPFC, section 5 describes the proposed CSA algorithm; section 6 explains the simulation results and discussion; and finally, conclusion is discussed in section 7 .

\section{Model of IPFC}

The basic schematic of IPFC is shown in Fig. 1. It consists of at least two back-to-back DC-AC converters connected through a common DC link and the DC link between each VSC can be represented by bidirectional link, for exchanging active power between them [19, 20]. The bus i,j and $\mathrm{k}$ has the complex voltages $\mathrm{V}_{\mathrm{i}}, \mathrm{V}_{\mathrm{j}}$, and $\mathrm{V}_{\mathrm{k}}$ and the series compensation of series converter voltage, $\mathrm{Vse}_{\mathrm{in}}$ is the controllable series injected voltage source which can be defined as $V_{s e_{i n}}=V_{s e_{i n}} \angle \theta_{s e_{i n}}(\mathrm{n}=\mathrm{j}, \mathrm{k})$. Fig. 1 consists of three buses $\mathrm{i}, \mathrm{j}$ and $\mathrm{k}$ and two transmission lines are connected with $\mathrm{i}^{\text {th }}$ bus in common. The equivalent circuit of two converters IPFC is shown in Fig. 2. It has two series injected voltages (Vse) and series with thr transfer impedance $\mathrm{Zse}_{\mathrm{in}}$. Pi and Qi are the sum of real and reactive power flow from $i^{\text {th }}$ bus is given in (1) and (2).

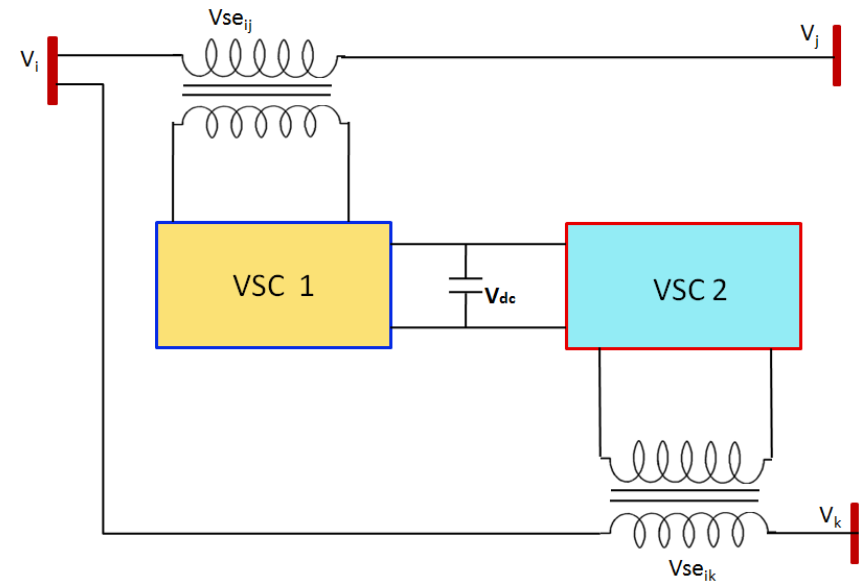

Fig. 1. Basic schematic of IPFC.

$\mathrm{P}_{\mathrm{i}}=\mathrm{V}_{\mathrm{i}}^{2} g_{i i}-\sum_{n} \mathrm{~V}_{\mathrm{i}} \mathrm{V}_{\mathrm{n}}\left(g_{i n} \theta_{\mathrm{i}}+b_{i n} \theta_{j}\right)-\sum_{n} \mathrm{~V}_{\mathrm{i}} \mathrm{V}_{\mathrm{se}_{\mathrm{in}}}\left(g_{i n} \theta_{\mathrm{k}}+b_{i n} \theta_{m}\right)$

$\mathrm{Q}_{\mathrm{i}}=-\mathrm{V}_{\mathrm{i}}^{2} \mathrm{~b}_{\mathrm{ii}}-\sum_{n=j, k} \mathrm{~V}_{\mathrm{i}} \mathrm{V}_{\mathrm{n}}\left(\mathrm{g}_{\mathrm{in}} \theta_{\mathrm{j}}-\mathrm{b}_{\mathrm{in}} \theta_{\mathrm{i}}\right)-\sum_{n=j, k} \mathrm{~V}_{\mathrm{i}} \mathrm{V}_{\text {se }}\left(\mathrm{g}_{\mathrm{in}} \theta_{\mathrm{m}}-\mathrm{b}_{\mathrm{in}} \theta_{\mathrm{k}}\right)$

Where $\theta_{i}=\cos \left(\theta_{i}-\theta_{n}\right), \quad \theta_{j}=\sin \left(\theta_{i}-\theta_{n}\right), \quad \theta_{k}=\cos \left(\theta_{i}-\theta_{s e_{i n}}\right), \quad \theta_{k}=\cos \left(\theta_{i}-\theta_{s e_{i n}}\right), \quad \theta_{m}=\sin \left(\theta_{i}-\theta_{s e_{i n}}\right)$, $\mathrm{n}=\mathrm{j} . \mathrm{k}$.

Similarly the real and reactive power flow from $\mathrm{n}^{\text {th }}$ bus, $\mathrm{P}_{\mathrm{ni}}$ and $\mathrm{Q}_{\mathrm{ni}}$ can be expressed as:

$$
\begin{aligned}
& \mathrm{P}_{\mathrm{ni}}=\mathrm{V}_{\mathrm{n}}^{2} g_{n n}-\mathrm{V}_{\mathrm{i}} \mathrm{V}_{\mathrm{n}}\left[g_{i n} \cos \left(\theta_{\mathrm{n}}-\theta_{\mathrm{i}}\right)+b_{i n} \sin \left(\theta_{n}-\theta_{\mathrm{i}}\right)\right]+V_{n} V_{s e_{i n}}\left\lfloor g_{i n} \cos \left(\theta_{\mathrm{n}}-\theta_{\mathrm{se}_{\mathrm{in}}}\right)-b_{i n} \sin \left(\theta_{n}-\theta_{\mathrm{se}_{\mathrm{in}}}\right)\right\rfloor \\
& \mathrm{Q}_{\mathrm{ni}}=-\mathrm{V}_{\mathrm{n}}^{2} b_{n n}-\mathrm{V}_{\mathrm{i}} \mathrm{V}_{\mathrm{n}}\left[g_{i n} \sin \left(\theta_{\mathrm{n}}-\theta_{\mathrm{i}}\right)-b_{i n} \cos \left(\theta_{n}-\theta_{\mathrm{i}}\right)\right]+V_{n} V_{s e_{i n}}\left\lfloor g_{i n} \sin \left(\theta_{\mathrm{n}}-\theta_{\mathrm{se}_{\mathrm{in}}}\right)-b_{i n} \cos \left(\theta_{n}-\theta_{\mathrm{se}_{\mathrm{in}}}\right)\right\rfloor
\end{aligned}
$$

According to the principle operation of IPFC [19] the active power can be exchanged between VSCs through DC link is given as:

$\mathrm{P}_{\text {sum }}=\sum_{\mathrm{j}=1, \mathrm{j} \neq \mathrm{i}}^{\mathrm{n}}\left\{\operatorname{Re}\left(\mathrm{V}_{\mathrm{se}_{\mathrm{ij}}} \mathrm{I}_{\mathrm{ij}}\right)\right\}=0$ 
Where $g_{i n}+j b_{i n}=1 / Z_{s e_{i n}}, g_{i i}=\sum_{n=j, k} g_{i n}, b_{i i}=\sum_{n=j, k} b_{i n}, g_{n n}+j b_{n n}=1 / Z_{s e_{i n}}$

III. Optimal Sizing of IPFC

The optimal sizing of IPFC minimizes load voltage deviation (LVD), active power loss and total capacity of installed IPFC. Optimal sizing is determined by formulating an objective function.

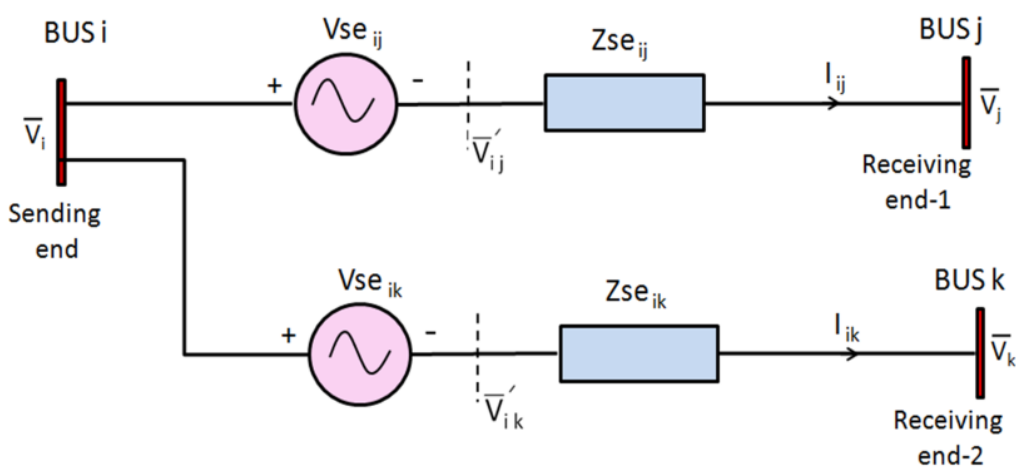

Fig. 2. Equivalent circuit of IPFC.

\subsection{Objective Function}

The multi objective function can be described as:

$\min F(x)=\min \sum_{i=1}^{3} f_{i}(x) w_{i}$

Where $\mathrm{w}_{\mathrm{i}}$ is the weight factor of individual objective function, $\mathrm{i}=1, \ldots, 3$. The weighting factor is used in the objective function to replicate its relative importance. In this work, all the individual weighting factors are considered as equal. So that $\mathrm{w}_{1}+\mathrm{w}_{2}+\mathrm{w}_{3}=1$.

\subsubsection{Minimization of load voltage deviation}

The voltage deviation at each bus should be as small as possible and the LVD for each bus can be expressed as [17]:

$\mathrm{f}_{1}(x)=\min \left(\sum_{i=1}^{\mathrm{n} \text { bus }}\left|V_{i}-V_{i}^{r e f}\right|^{2}\right)$

where $\mathrm{V}_{\mathrm{i}}$ magnitude of voltage at bus $\mathrm{i}$.

\subsubsection{Active power loss reduction}

The first objective function is the active power loss reduction and it can be applied based on [13] as:

$$
\min \mathrm{f}_{1}(x)=\sum_{j=1}^{m} P_{G j}-\sum_{i=1}^{n} P_{L i}
$$

where $\mathrm{P}_{\mathrm{Gj}}$ is the active power generated by the $\mathrm{j}$-th generator with the constraint $P_{G j \min } \leq P_{G j} \leq P_{G j m i a x}$ and $\mathrm{P}_{\mathrm{Li}}$ is the active power consumed by the i-th load bus with the constraint $P_{L i \text { min }} \leq P_{L i} \leq P_{L i \max }$.

\subsubsection{Maximization of VSM}

The Maximum Loadability Index (MLI) define the voltage stability margin. The risk of voltage collapse can be reduced by increasing the value of MLI and it leads to increase the voltage stability margin. The MLI can be determined based on [39] and it can be expressed as:

$$
M L I_{k}=\frac{V_{j}^{2}}{\left.2\left(\left[P_{j k} R_{j k}+X_{j k} Q_{j k}\right]+\sqrt{\left[R_{j k}^{2}+X_{j k}^{2}\right] P_{j k}^{2}+X_{j k}^{2}}\right]\right)}
$$

where $R_{j k}$ is the resistance and $X_{j k}$ is the reactance between the bus $j$ and $k . P_{j k}$ and $Q_{j k}$ are the real and reactive power flow between the bus $\mathrm{j}$ and $\mathrm{k}$ respectively. When load increases, the value of MLI decreases and the third objective function can be expressed as:

$\mathrm{f}_{3}(x)=\max \left(\sum_{i=1}^{N} M L I(i)\right)$

where $\mathrm{N}$ is the number of buses. 


\subsubsection{Minimization of total capacity of IPFC}

It is necessary to determine the total capacity for solving overloading of line. The total capacity of placed IPFC in the optimal location can be expressed by the multi-objective function as [21]:

$$
\begin{aligned}
& \mathrm{f}_{4}(x)=\min \left(P Q_{1}^{2}+P Q_{2}^{2}\right) \\
& P Q_{1}^{2}+P Q_{2}^{2}=\left(V s e_{i j}\left(\frac{\overline{V_{i}-V s e_{i j}-V_{j}}}{Z_{i j}}\right)\right)^{2}+\left(V s e_{i k}\left(\frac{\overline{V_{i}-V s e_{i k}-V_{k}}}{Z_{i k}}\right)\right)^{2}
\end{aligned}
$$

where PQ is the capacity of each VSCs of IPFC. The eqn. (12) is subject to various constraints given in the following sub section.

\subsection{Contingency Constraints}

The main objective of this study is to determine the optimal location of IPFC and congestion management for enhancing the power system security. Contingency event occurs if the outage of a generator or transmission line becomes unstable. The preventive and/or corrective action should be taken before the transmission line enters into an insecure state. Contingency analysis is one of the important functions to solve this state. In this paper, we considered multiline outage contingencies for IEEE 30-bus test system. We considered a voltage limit violation present in the buses; overloaded lines and flow limit for determine each line contingency. The constraints are:

Voltage constraints: $\mathrm{V}_{\mathrm{i}}^{\mathrm{min}} \leq \mathrm{V}_{\mathrm{i}} \leq \mathrm{V}_{\mathrm{i}}^{\mathrm{max}} ; \forall \mathrm{i} \in$ loadbus

Line flow limit: $\mathrm{F}_{\mathrm{k}}^{\text {min }} \leq \mathrm{F}_{\mathrm{k}} \leq \mathrm{F}_{\mathrm{k}}^{\max } ; \mathrm{k} \in \mathrm{N}$

Power generation limit: $\mathrm{P}_{\mathrm{ij}}^{\min } \leq \mathrm{P}_{\mathrm{ij}} \leq \mathrm{P}_{\mathrm{ij}}^{\max } ; \forall \mathrm{ij}$

IPFC constraints: $\mathrm{V}_{\mathrm{se}}^{\min } \leq \mathrm{V}_{\mathrm{se}} \leq \mathrm{V}_{\mathrm{se}}^{\max }$ and $\theta_{\mathrm{se}}^{\min } \leq \theta_{\mathrm{se}} \leq \theta_{\mathrm{se}}^{\max }$

After finding the violations, the severity order is allotted for the lines. The CSA is applied for the critical contingency and to find out the optimized location and other parameters of IPFC. Installing IPFC in the optimized location is determined by CSA which is used to eliminate or minimize the overloading and bus voltage violation limits. The real and reactive power flow constraints can be taken from equation 1 and 2 .

\section{Optimal Allocation of IPFC}

LUF is used to determine the percentage of utilization of a line and estimate the congestion efficiently [21]. It can be expressed as:

$$
L U F_{i j}=\frac{M_{i j}}{M_{i j}}
$$

where LUF $_{\mathrm{ij}}$, MVAij, MVAijmax are the LUF of the line connected to bus $\mathrm{i}$ and $\mathrm{j}$, MVA rating of the line between bus $i$ and bus $j$, maximum MVA rating of the line between bus $i$ and $j$ respectively. LUF is not sufficient for placing the IPFC because it is required that at least two lines should be connected to a common bus. Hence another approach is proposed for optimal placement of IPFC. In this approach the difference between utilization of lines (SLUF) are considered and it provides the difference in the percentage of line being utilized for the power flow. SLUF specify that there is a possibility of active power exchange between the IPFC converters as a result exact allocation of IPFC is done. SLUF can be expressed as:

$$
S L \mathrm{UF}_{(\mathrm{ij})-(\mathrm{ik})}=\left|\frac{\mathrm{MVA}_{\mathrm{ij}}-\mathrm{MVA}_{\mathrm{ik}}}{\mathrm{MVA}_{\max }}\right|
$$

where $\mathrm{SLUF}_{(\mathrm{ij})-(\mathrm{ik})}, \mathrm{MVA}_{\mathrm{ij}}, \mathrm{MVA}_{\mathrm{ik}}, \mathrm{MVA}_{\max }$ are the SLUF of the line set i-j and i-k, MVA rating of the line between bus $i$ and bus $j$, MVA rating of the line between bus $i$ and bus $k$, and the maximum MVA rating of the lines connected between bus $i$ and bus $j$ respectively.

\section{Overview of CSA}

CSA obliges brood parasitism of cuckoo species by laying their eggs in the host birds' nests. CSA is a stochastic global search meta-heuristics with random walk based on population. This algorithm is a mixture with the Levy flight behavior of some birds [22] and motivated us to calculate the location and other parameters such as overloaded lines, voltage violation limit, and congestion management of IPFC. CSA follows three important rules which are: 1) each cuckoo can choose the location randomly and lay their eggs one at a time. 2) The highest quality eggs present in their nest are founded by Elitist selection process and carry over to next 
generation. 3) The host nest number cannot be adjustable and the egg laid by the cuckoo can be founded by the probability $\mathrm{P}_{\mathrm{d}} \varepsilon(0,1)$ proposed in [23].

At first, the objective function value $\mathrm{F}_{\mathrm{i}}(\mathrm{x})$ for the $\mathrm{i}$-th population is $F_{i}(x)=\mathrm{f}\left(\mathrm{x}_{1 \mathrm{i}}, \mathrm{x}_{2 \mathrm{i}}, \mathrm{x}_{3 \mathrm{i}}, \ldots, \mathrm{x}_{\mathrm{ni}}\right), \forall_{\mathrm{i}}=1,2, \ldots, \mathrm{n}$. where $\mathrm{n}$ is the number of control variables and $\mathrm{m}$ is the total number of populations. It can be expressed as:

$\left[\begin{array}{c}F_{1}(x) \\ F_{2}(x) \\ : \\ F_{n}(x)\end{array}\right]=\left[\begin{array}{c}f\left(x_{11}, x_{21}, \ldots, x_{n 1}\right) \\ f\left(x_{12}, x_{22}, \ldots, x_{n 2}\right) \\ : \\ f\left(x_{1 m}, x_{2 m}, \ldots, x_{m n}\right)\end{array}\right]$

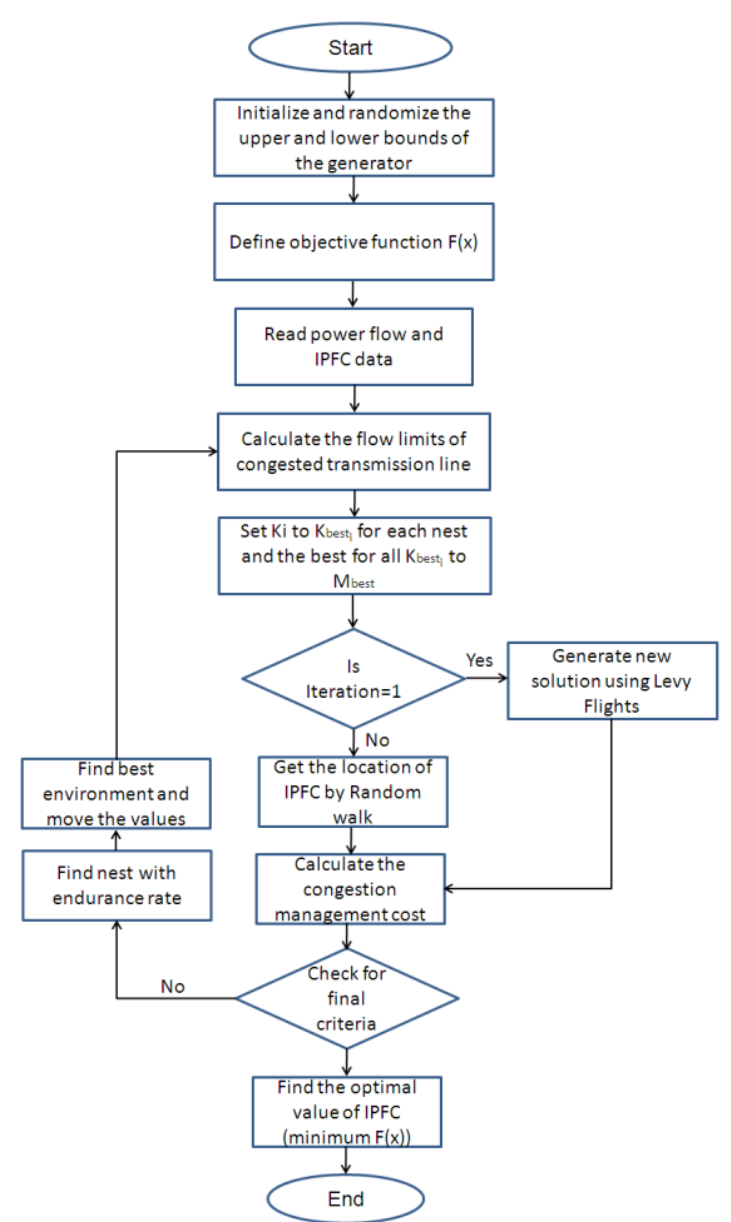

Fig. 3. Flowchart of the implemented CSA algorithm.

The size of population of the each control variable can be randomly generated as:

$x_{i j}=x_{\mathrm{i}}^{\min }+\operatorname{rand}(0,1) \times\left(x_{j}^{\max }-x_{j}^{\min }\right)$

where $i$ is the number of host nests; $i=1,2,3, \ldots ., n$ and $j$ is the number of control variables; $j=1,2,3, \ldots, m$.

$x_{\mathrm{i}}^{\min }$ and $x_{\mathrm{i}}^{\max }$ are the lower and upper limits of $\mathrm{i}$-th control variable respectively. rand $(0,1)$ is uniformly distributed and the solution of parameter estimation is feasible because they are initialized within the feasible range and we have to find the optimal one. For generating new solutions levy flight is performed [23]. For calculating the location and setting other parameters of IPFC we have chosen simple scheme where each nest has only one egg. The Cuckoo, $\mathrm{K}$ generates a new solution $\mathrm{X}_{\mathrm{k}}{ }^{(t+1)} \mathrm{using}$ Levy flight is based on the following equation:

$x_{i}(k+1)=x_{i}(k)+S Z_{i j} \times \beta \oplus \operatorname{lev} y(\lambda)$

Where $\mathrm{SZ}_{\mathrm{ij}}$ is the step size $\left(S Z_{i j}=x_{\mathrm{ij}}^{\mathrm{k}}-x_{\mathrm{fj}}^{\mathrm{k}}\right), \beta$ represents the step size and we assume that the step size is 1 and $\otimes$ represents the entry wise multiplication. Equation (17) shows that the stochastic equation for random walk. Random walk is based on Markov chain whose next status depends on the current location and the transition probability. CSA has high speed of convergence compared to other optimization techniques such as 
PSO and DEA. The success rate and efficiency is also high in CSA. In this paper, a continuous, multi objective optimization problem has been considered for optimal tuning of IPFC and congestion management. Based on the three rules, the optimal tuning using CSA has been given in the flowchart as shown in Fig. 3.

The fitness value of the radial topology can be calculated using eq. (8). In the simulation the common control parameters such as maximum generation and number of cuckoo are considered as 50 and 20 respectively. The minimum values less than $10^{-6}$ are not included and assumed to be zero. On the other hand the algorithmic control of PSO is set according to [24] and the values $\mathrm{C}_{1}=\mathrm{C}_{2}=1.8$, and $\omega=0.6$ have been used. The generating cuckoos and alignment steps are alternatively performed until it reaches the maximum iteration $\left(\mathrm{It}_{\max }\right)$.

\section{Results and Discussion}

To demonstrate the performance and effectiveness of the proposed CSA technique, the standard IEEE 30-bus test systems is considered and the result is compared with two optimization techniques such as PSO and DEA. The simulation was developed using MATLAB R2014a in Intel core i5, $2.3 \mathrm{GHz}$ with 6 GB RAM personal computer. The lower voltage limit $\left(\mathrm{V}_{\min }=0.95 \mathrm{p} . \mathrm{u}\right)$, the upper voltage limit $\left(\mathrm{V}_{\max }=1 \mathrm{p} . \mathrm{u}\right)$, and the threshold value of power flow analysis is 0.006 has been set. The voltage magnitude and angle of two converters of IPFC is taken in the range $0 \leq \mathrm{V}_{\mathrm{se}} \leq 1$ and $-\pi \leq \theta_{\mathrm{se}} \leq \pi$ respectively. In IEEE 30-bus system, bus number 4 is a slack bus, bus numbers $1,2,8,9$, and 11 are considered as PV buses and all other buses are considered as load buses. The single line diagram of IEEE 30-bus system with IPFC between buses 25-29 and 29-30 is shown in Fig. 4. The network topology and data for simulating above systems are taken from the University of Washington [25]. The performances of CSA, PSO and DE algorithms are studied together for comparison. Different initial parameters have been used for implementing CSA, PSO and DEA to find the optimal values of IPFC are presented in Table 1.

\subsection{Optimal allocation of IPFC}

To demonstrate the performance of the proposed CSA technique, a large scale of distributed network with 30 nodes are considered. The initial minimum bus voltage was 0.95 p.u and total load power is $21953.4 \mathrm{~W}$. The series inductive reactance is $2.73 \%$ p.u. The coupled pi-section inductive reactance is $5.79 \%$ p.u, the susceptance is $5.62 \%$ p.u, and resistance is $1.89 \%$ p.u. The series coupling transformer has 30.32 MVA with leakage reactance is $1.0 \%$ p.u, and has a winding ratio of $22.73 \mathrm{kV} / 9.21 \mathrm{kV}$.

The voltage limit range of $0.95-1.1$ P.U is considered for (easy calculation) determining voltage violation and $100 \%$ loading is considered for determining overloaded lines. The total overloaded lines (TOL), total violation buses (TVB), and the priority of the line is presented in Table 2. Based on these considerations 14 lines comes under severe contingency scenarios. From Table 2 one can easily understand that the line connected between 4 to 5 is most congested lines between the load buses. Hence, the first and second converter of IPFC can be inserted between the lines 25-29 and the line has a bus common with line 25-30respectively.

Table 1. Initialization parameters for implementing CSA, PSO and DEA techniques.

\begin{tabular}{|c|c|c|c|c|c|}
\hline CSA Technique & Value & PSO Technique & Value & DEA Technique & Value \\
\hline Number of cuckoos & 50 & Number of swam & 50 & $\begin{array}{l}\text { Maximum number of } \\
\text { generations }\end{array}$ & 400 \\
\hline $\begin{array}{l}\text { Maximum number } \\
\text { of generations }\end{array}$ & 400 & $\begin{array}{l}\text { Maximum number } \\
\text { of flights }\end{array}$ & 400 & Mutation Rate & 0.2 \\
\hline $\begin{array}{l}\text { Minimum number } \\
\text { of eggs for each } \\
\text { cuckoo }\end{array}$ & 20 & Wmax & 0.9 & $\begin{array}{l}\text { Fraction of population } \\
\text { kept } \\
\text { Crossover rate }\end{array}$ & $\begin{array}{l}0.5 \\
0.2\end{array}$ \\
\hline $\begin{array}{l}\text { Maximum number } \\
\text { of eggs for each } \\
\text { cuckoo }\end{array}$ & 40 & Wmin & 0.4 & Number of variables & 15 \\
\hline Motion coefficient & 9 & $\begin{array}{l}\text { Termination } \\
\text { criteria }\end{array}$ & 1.exp-6 & & \\
\hline Number of cluster & 1 & Weighting factors & $\begin{array}{l}c_{1}=2, \\
c_{2}=1.5\end{array}$ & & \\
\hline Termination criteria & 1.exp-6 & Deviation & & & \\
\hline
\end{tabular}




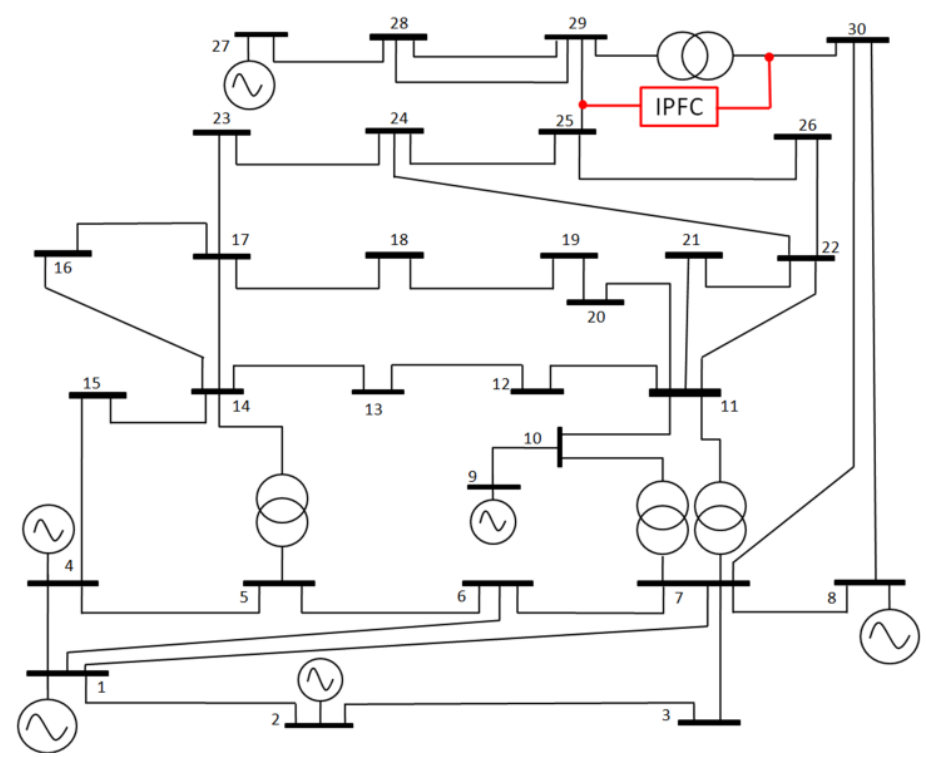

Fig. 4. Single line diagram of IEEE 30-bus system with IPFC placed between buses 25-29 and 29-30.

Table 2. Contingency analysis and priority fixing for IEEE 30-bus system.

\begin{tabular}{ccccccc}
\hline \multicolumn{6}{c}{ Tripped Lines } & \multicolumn{2}{c}{$\begin{array}{l}\text { Total } \\
\text { overloaded } \\
\text { Line }\end{array}$} & $\begin{array}{l}\text { From } \\
\text { Number }\end{array}$ & $\begin{array}{l}\text { To } \\
\text { bus } \\
\text { bus }\end{array}$ & $\begin{array}{l}\text { Total } \\
\text { voltage } \\
\text { violation } \\
\text { buses } \\
\text { (TVB) }\end{array}$ & $\begin{array}{c}\text { TOL } \\
\text { TVB }\end{array}$ & Priority \\
\hline 2 & 1 & 2 & 6 & 5 & 11 & 6 \\
4 & 3 & 5 & 6 & 3 & 9 & 10 \\
5 & 2 & 6 & 7 & 5 & 12 & 5 \\
8 & 12 & 15 & 1 & 4 & 5 & 15 \\
9 & 3 & 5 & 2 & 2 & 4 & 17 \\
10 & 3 & 4 & 5 & 4 & 9 & 9 \\
11 & 7 & 10 & 5 & 3 & 8 & 11 \\
14 & 3 & 6 & 3 & 5 & 8 & 12 \\
21 & 14 & 17 & 7 & 3 & 10 & 8 \\
23 & 9 & 11 & 2 & 2 & 4 & 18 \\
24 & 6 & 8 & 5 & 6 & 11 & 7 \\
25 & 16 & 19 & 5 & 1 & 6 & 14 \\
27 & 25 & 27 & 7 & 6 & 13 & 3 \\
29 & 14 & 17 & 3 & 2 & 5 & 16 \\
\hline
\end{tabular}

\subsection{Optimal tuning of IPFC}

The voltage profile before after utilizing IPFC for IEEE 30-bus system is shown in Fig. 5. It is observed that after the placement of IPFC the bus voltage of the system has improved significantly.

The multi objective function $\mathrm{F}(\mathrm{x})$ is used to tune the IPFC and the values are shown in Fig. 6. From Fig. 6 one can easily understand that the number of cuckoo and number of generation increases, the value of objective function decreases. Hence, to minimize computation time, the number of cuckoo can be chosen 50 and number of generation in the range 100 to 400 . Comparison of power loss, VD, and capacity of inserted IPFC has been performed for different cases such as un-tuned IPFC, IPFC with PSO-tuned, IPFC with DEA-tuned and IPFC with CSA-tuned under different loading condition are presented in Table 3. 


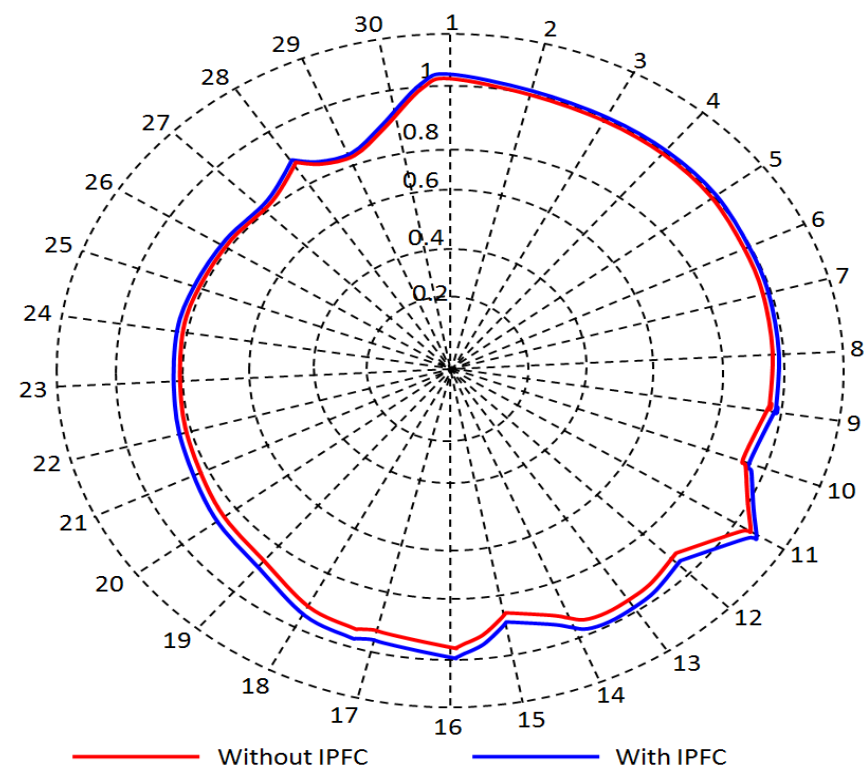

Fig. 5. Comparison of voltage profile with and without IPFC.

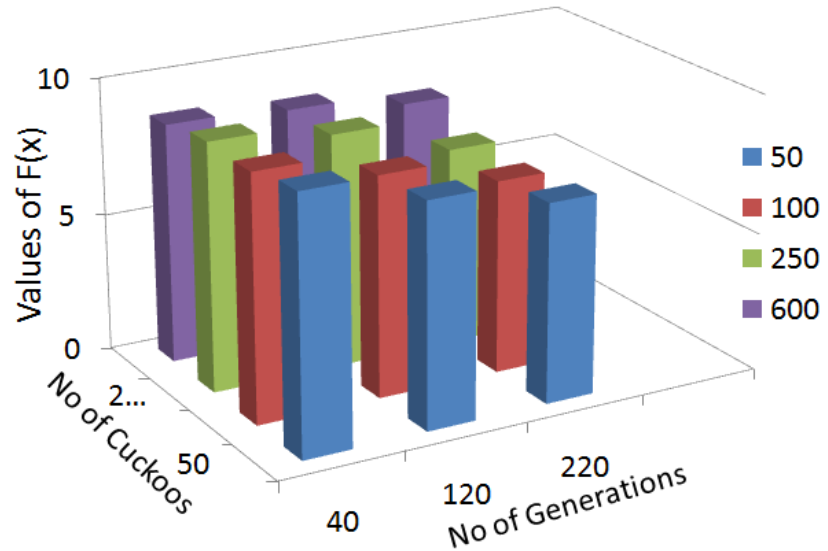

Fig. 6. Objective function value for various parameter setting of the CSA.

Table 3. Comparison of objective function values for un-tuned, tuned IPFC using PSO, DEA and CSA under different loading condition.

\begin{tabular}{|c|c|c|c|c|c|c|c|c|c|c|c|c|}
\hline \multirow[t]{2}{*}{ Parameters } & \multicolumn{4}{|c|}{ Normal loading } & \multicolumn{4}{|c|}{$110 \%$ loading } & \multicolumn{4}{|c|}{$120 \%$ loading } \\
\hline & $\begin{array}{l}\text { Untuned } \\
\text { IPFC }\end{array}$ & $\begin{array}{l}\text { Tuned } \\
\text { IPFC using } \\
\text { PSO }\end{array}$ & $\begin{array}{l}\text { Tuned } \\
\text { IPFC using } \\
\text { DEA }\end{array}$ & $\begin{array}{l}\text { Tuned } \\
\text { IPFC using } \\
\text { CSA }\end{array}$ & $\begin{array}{l}\text { Untuned } \\
\text { IPFC }\end{array}$ & $\begin{array}{l}\text { Tuned } \\
\text { IPFC using } \\
\text { PSO }\end{array}$ & $\begin{array}{l}\text { Tuned } \\
\text { IPFC using } \\
\text { DEA }\end{array}$ & $\begin{array}{l}\text { Tuned } \\
\text { IPFC using } \\
\text { CSA }\end{array}$ & $\begin{array}{l}\text { Untuned } \\
\text { IPFC }\end{array}$ & $\begin{array}{l}\text { Tuned } \\
\text { IPFC using } \\
\text { PSO }\end{array}$ & $\begin{array}{l}\text { Tuned } \\
\text { IPFC using } \\
\text { DEA }\end{array}$ & $\begin{array}{l}\text { Tuned } \\
\text { IPFC using } \\
\text { CSA }\end{array}$ \\
\hline Real power loss, MW & 22.429 & 21.845 & 21.703 & 21.203 & 27.374 & 27.045 & 26.935 & 25.528 & 34.15 & 33.924 & 33.920 & 32.717 \\
\hline VD of all buses, pu & 2.5380 & 2.5234 & 2.5257 & 2.5125 & 2.5859 & 2.5684 & 2.5698 & 2.1053 & 2.6267 & 2.6015 & 2.6002 & 2.5623 \\
\hline SM of all lines, pu & 19.5951 & 16.3754 & 16.3483 & 16.3261 & 19.7369 & 16.7350 & 16.6943 & 16.1053 & 21.3842 & 18.2781 & 18.2798 & 18.1634 \\
\hline $\begin{array}{l}\text { Total capacity of } \\
\text { installed IPFC, pu }\end{array}$ & 0.000582 & 0.0000063 & 0.0000061 & 0.0000060 & 0.000253 & 0.000217 & 0.000213 & 0.000182 & 0.000240 & 0.000209 & 0.000203 & 0.000172 \\
\hline
\end{tabular}

Table 4. Real and reactive power loss with and without IPFC, tuned IPFC using PSO, DEA and CSA for IEEE 30-bus test system under different loading condition.

\begin{tabular}{|c|c|c|c|c|c|c|}
\hline \multirow[t]{2}{*}{ Condition } & \multicolumn{2}{|c|}{ Normal loading } & \multicolumn{2}{|c|}{$110 \%$ loading } & \multicolumn{2}{|c|}{$120 \%$ loading } \\
\hline & $\begin{array}{l}\text { Real power } \\
\text { losses, } \\
\text { MW }\end{array}$ & $\begin{array}{l}\text { Reactive } \\
\text { power } \\
\text { losses, } \\
\text { MVAR }\end{array}$ & $\begin{array}{l}\text { Real } \\
\text { power } \\
\text { losses, } \\
\text { MW }\end{array}$ & $\begin{array}{l}\text { Reactive } \\
\text { power } \\
\text { losses, } \\
\text { MVAR }\end{array}$ & $\begin{array}{l}\text { Real power } \\
\text { losses, } \\
\text { MW }\end{array}$ & $\begin{array}{l}\text { Reactive } \\
\text { power } \\
\text { losses, } \\
\text { MVAR }\end{array}$ \\
\hline Without IPFC & 23.143 & 108.539 & 28.552 & 128.583 & 37.537 & 163.367 \\
\hline Untuned IPFC & 21.845 & 102.627 & 27.395 & 119.459 & 35.628 & 149.936 \\
\hline Tuned IPFC using PSO & 21.6386 & 101.238 & 27.021 & 118.158 & 34.137 & 149.027 \\
\hline Tuned IPFC using DEA & 21.5279 & 100.927 & 26.925 & 118.118 & 33.943 & 148.847 \\
\hline Tunes IPFC using CSA & 21.1205 & 99.364 & 24.374 & 115.175 & 30.264 & 148.035 \\
\hline
\end{tabular}


The voltage deviation is reduced from 2.53 to 2.31 pu after inserting the IPFC at the optimal location. It is noted that after placing IPFC at the optimal location, the congestion in line 4 and 5 has been reduced from 0.8524 to 0.8382 . The real and reactive power loss of the system with and without IPFC and optimally tuned IPFC with PSO, DEA and CSA under different loading conditions is presented in Table 4. It is observed from Table 4 that the real and reactive power loss is very less using CSA compared with PSO and DEA for all the loading conditions.

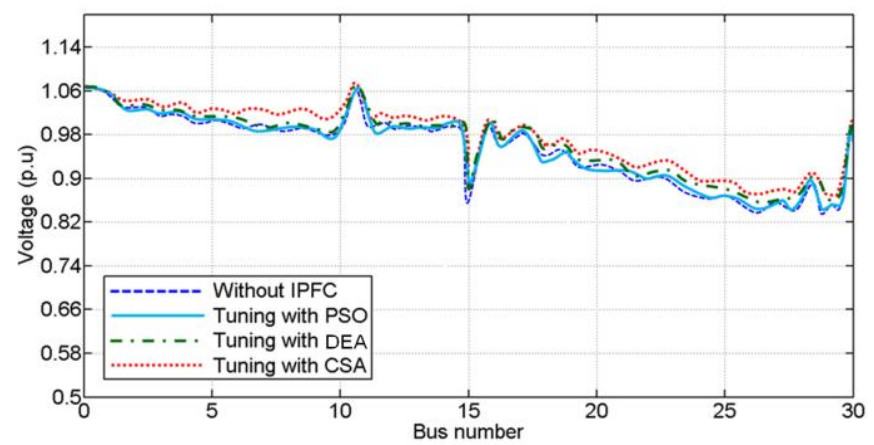

Fig. 7. Comparison of voltage profile without IPFC and tuned IPFC using CSA, PSO and DEA for IEEE 30-bus test system under normal loading condition.

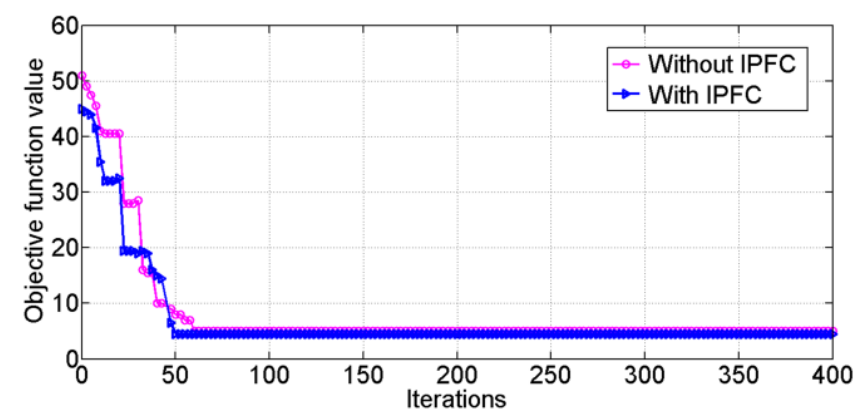

Fig. 8. Convergence characteristics with and without IPFC under normal loading condition.

\subsection{Results for various loading}

The voltage profile for the buses after tuning of IPFC using PSO, DEA and CSA under normal loading condition is shown in Fig. 7. It is noted from Fig. 7 that the tuning of IPFC reduces VD, reduction in loss helps in congestion management of the system and reduction in SM protects the system against collapse. The convergence results of with and without IPFC under normal loading condition is shown in Fig. 8. The minimum fitness after 106 iterations. Even the initial population set of all the algorithms are equal, the CSA initiate the best solution with the fitness value of 1.031 at the first iteration because CSA generates two solution sets through Levy flights and alignment of eggs. On the other hand, PSO and DEA have the fitness value of 1.106 and 1.091 respectively.

The load is increased to $110 \%$ and the simulation is carried out on IEEE 30-bus system. It is noted that when load increase the real and reactive power losses increases. From Table 3 and 4 one can easily understand that optimal placement of IPFC with tuning reduces the loss, VD and capacity of installed IPFC. The voltage profile of 30-bus system without IPFC and IPFC tuned with CSA, PSO and DEA are shown in Fig. 9. It is observed from Fig. 9 that optimally tuned IPFC performs better than without IPFC. On comparing CSA with PSO and DEA, the CSA method performs better than other two methods. The convergence results of CSA, PSO and DEA under $110 \%$ loading condition are shown in Fig. 10. It is noted that, after 32, 50 and 122 iterations, the CSA, DEA and PSO attain the minimum fitness respectively. The load is increased to $120 \%$ and the simulation is carried out on IEEE 30-bus system is shown in Fig. 11. It is observed from Fig. 11 that optimally tuned IPFC performs better than without IPFC. 


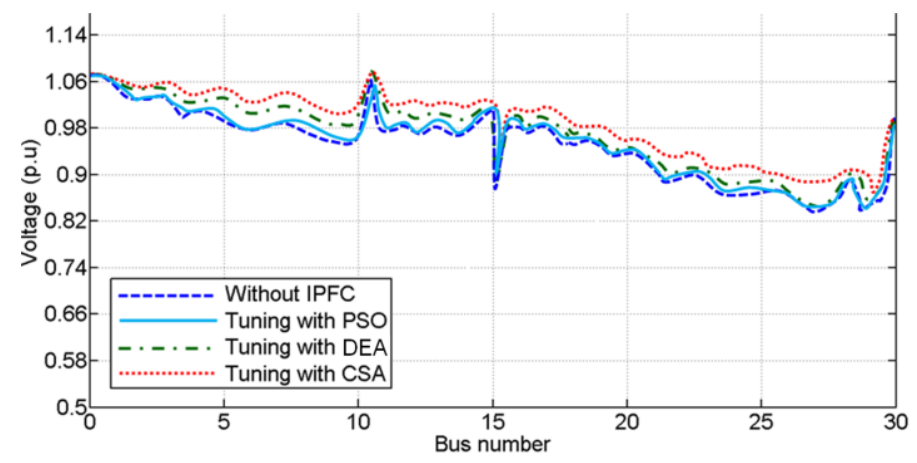

Fig. 9. Comparison of voltage profile without IPFC and tuned IPFC using CSA, PSO and DEA for IEEE 30-bus

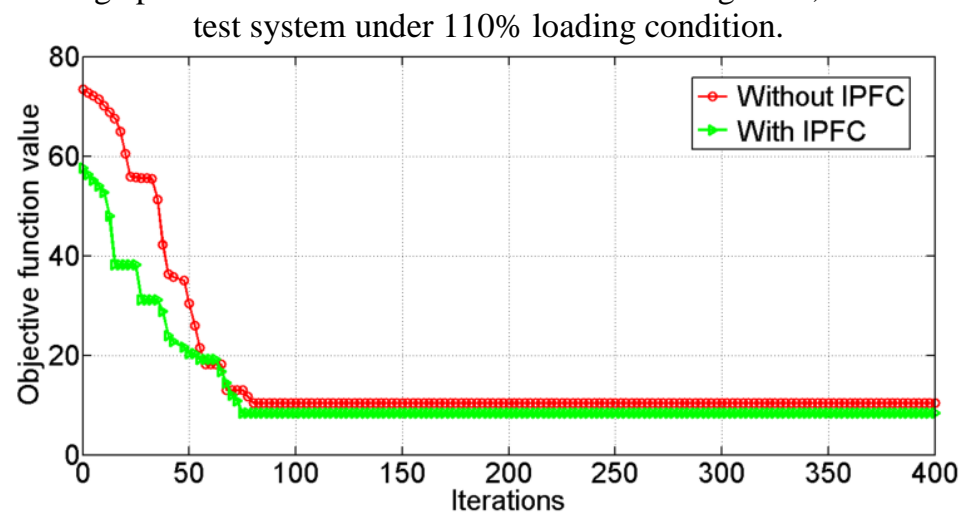

Fig. 10. Convergence characteristics with and without IPFC under $110 \%$ loading condition.

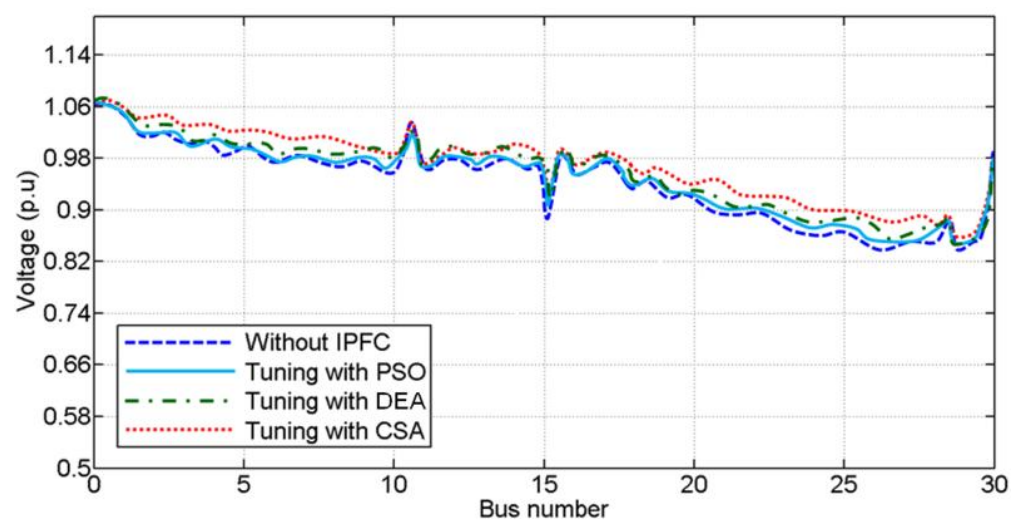

Fig. 11. Comparison of voltage profile without IPFC and tuned IPFC using CSA, PSO and DEA for IEEE 30bus test system under $120 \%$ loading condition.

On comparing CSA with PSO and DEA, the CSA method performs better than other two methods. The convergence results of CSA, PSO and DEA under 120\% loading condition are shown in Fig. 12. It is noted that, after 40, 63 and 140 iterations, the CSA, DEA and PSO attain the minimum fitness respectively.

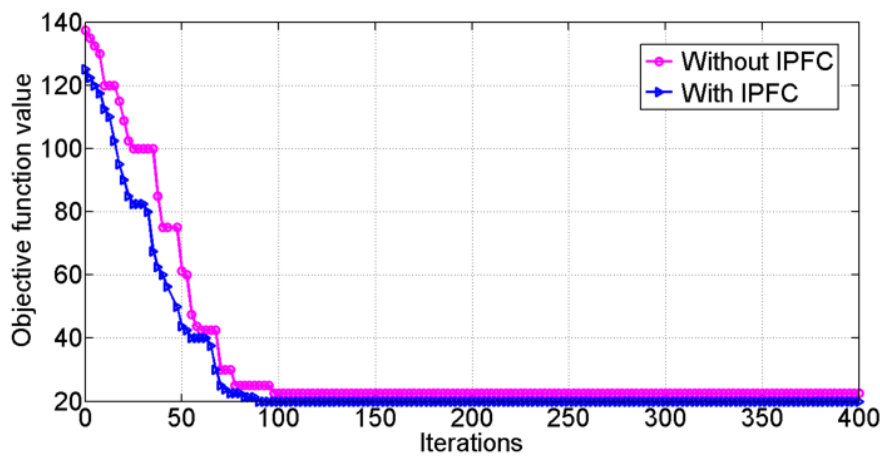

Fig. 12. Convergence characteristics with and without IPFC under $120 \%$ loading condition. 


\section{Conclusion}

In this paper a new method is proposed for proper placement of IPFC based on cuckoo search algorithm. Subtraction of line utilization factor is proposed for optimal placement of IPFC for congestion management. The IPFC is placed according to the value of SLUF with optimal tuning using CSA. Before inserting the IPFC in the optimal location, the percentage of overloading of some line is very high which leads to trip the line and continuous failure in the system and nearby system as well. After utilizing IPFC in the optimal locations, voltage violations are eliminated and overloading is reduced with considerable amount. The performance of CSA is done using IEEE 30-bus test systems and is compared with two other optimization techniques such as PSO and DEA and the results show that the effectiveness of CSA for tuning of IPFC. The multi objective function is formulated and tuned using CSA and the performance shows that tuning of IPFC reduces the real power loss, and voltage violation of transmission lines. In addition, optimal tuning much reduces the capacity of installed IPFC. It is also noted that the performance of the system improved significantly with IPFC.

\section{References}

[1] Masoud Mohammadalizadeh-Shabestary, Hamed Hashemi-Dezaki, Shahed Mortazavian, Hossein Askarian-Abyaneh, and Gevork Gharehpetian, A general approach for optimal allocation of FACTS devices using equivalent impedance models of VSCs, International Transactions on Electrical Energy Systems, 25(7), 2014, 307-1203.

[2] Masahide Hojo, Yasunori Mitani, Toshifumi Ise, and Kiichiro Tsuji, Quantitative evaluation of generator power control effect of FACTS controllers for power system stabilization, Electrical engineering in Japan, 138, 2002, 43-51.

[3] Kalyan K. Sen, Analysis of facts controllers and their transient modelling techniques, John Wiley \& Sons, Ltd 2015

[4] Wei Xuan, Chow Joe H, Fardanesh Behruz, Edris Abdel-Aty, A common modeling framework of voltage-sourced converters for load flow, sensitivity, and dispatch analysis, IEEE Transactions on Power System, 19(2), 2004, 934-941.

[5] Valentin Azbe, and Mihalic, The Control Strategy for an IPFC Based on the Energy Function, IEEE Transactions on Power Systems, 23(4), 2008, 1662-1669.

[6] E. Gholipour, GH, Isazadeh, Design of a New Adaptive Optimal Wide Area IPFC Damping Controller in Iran Transmission Network, Electrical Power and Energy Systems. 53, 2013, 529-539.

[7] Vedik Basetti, Ashwani K. Chandel, Hybrid power system state estimation using Taguchi differential evolution algorithm, IET Science, Measurement \& Technology, 9(4), 2015, 449-466.

[8] H. R. Cai, C. Y. Chung, K. P. Wong, Application of Differential Evolution Algorithm for Transient Stability Constrained Optimal Power Flow, IEEE Transactions on Power Systems.23(2), 2008, 719-722.

[9] Guang Ya Yang, Zhao Yang Dong, and Kit Po Wong, A Modified Differential Evolution Algorithm with Fitness Sharing for Power System Planning, IEEE Transactions on Power Systems. 2008;23(2):514-522.

[10] S. Hajforoosh S.M.H. Nabavi M.A.S. Masoum, Coordinated aggregated-based particle swarm optimization algorithm for congestion management in restructured power market by placement and sizing of unified power flow controller, IET Science, Measurement and Technology,6(4), 2012, 267-278.

[11] Sanjay Saini, Dayang Rohaya B, Awang Rambli, M. Nordin B. Zakaria, and Suziah B Sulaiman, A Review on Particle Swarm Optimization Algorithm and Its Variants to Human Motion Tracking, Hindawi Publishing Corporation. Mathematical Problems in Engineering, 2014, 116.

[12] Puja Dash, Lalit Chandra Saikia, Nidul Sinha, Comparison of performances of several FACTS devices using Cuckoo search algorithm optimized 2DOF controllers in multi-area AGC, Electrical Power and Energy Systems, 65, 2015, 316-324.

[13] Thuan Thanh Nguyen, Anh Viet Truong, Distribution network reconfiguration for power loss minimization and voltage profile improvement using cuckoo search algorithm, Electrical Power and Energy Systems, 68, 2015, 233-242.

[14] Abdelaziz A.Y, E.S. Ali, Cuckoo Search algorithm based load frequency controller design for nonlinear interconnected power system, Electrical Power and Energy Systems, 73, 2015, 632-643.

[15] Dieu N. V, Peter Schegner, Weerakorn Ongsakul, Cuckoo search algorithm for non-convex economic Dispatch, IET Generation, Transmission \& Distribution, 7(6), 2013, 645-654.

[16] Rasoul Azizipanah-Abarghooee, Taher Niknam, Mohsen Zare, Masihallah Gharibzadeh, Multi-objective short-term scheduling of thermoelectric power systems using a novel multi-objective $\theta$-improved cuckoo optimization algorithm, IET Generation, Transmission \& Distribution, 8(5), 2014, 873-894.

[17] Zhang, Y. and C. Chen, A novel power injection model of IPFC for power flow analysis inclusive of practical constraints, IEEE Transactions on Power Systems, 21, 2006, 1550-1556.

[18] Smt. Ushasurendra Parthssarthy S. S, Congesstion managent in deregulated power sector using fuzzy based optimal location technique for series flexible alternative current transmission system (FACTS) devices, J. Electr. Electron. Eng. Res., 4(1), 2012, 12-20.

[19] Hingorani, N. G. and L. Gyugyi, Understanding FACTS: concepts and technology of flexible AC transmission systems, Wiley-IEEE Press, 1999, 51-295.

[20] Farjah. E, Bornapour. M, Nikman. T, B. Bahmanifrouzi, Placement of combined heat, power and hydrogen production fuel cell power plant in a distribution network, Energies, 5, 2012, 790-814.

[21] B. Venkatesh, Rakesh Ranjan, H. B. Gooi, Optimal Reconfiguration of Radial Distribution Systems to Maximize Loadability, IEEE Trans. Power Systems, 19(1), 2004, 260- 266.

[22] Ramin Rajabioun, Cuckoo Optimization Algorithm, Applied soft computing, 11, 2011, 5508-5518.

[23] Yang Xin-She, and Deb Suash, Cuckoo Search via Levy flights. World Congress on Nature \& Biologically Inspired Computing, 2009, 210214.

[24] Eberhart RC, Shi Y, Particle Swam Optimization: development, applications and resources, IEEE Press. 2001, 90-283.

[25] Power Systems Test Case Archive, University of Washington, Seattle, 2013, Available online at: http:/ /www. ee. washington.edu/research/pstca/

[26] Chandrasekaran K, Simon SP, Multi-objective scheduling problem: hybrid approach using fuzzy assisted cuckoo search algorithm, Swarm Evol Comput., 5, 2012, 1-16.

[27] Gandomi A, Yang XS, Alavi A, Cuckoo search algorithm: a metaheuristic approach to solve structural optimization problems, Eng Comput., 29(1), 2013, 17-35. 